УДК 004.932

\title{
ХАРАКТЕРИСТИКИ ЭКГ-СИГНАЛА С ИСПОЛЬЗОВАНИЕМ ПОЛИНОМОВ ЛАГРАНЖА-ЧЕБЫШЕВА
}

\author{
О. П. ЯДАВ, Ш. РАЙ \\ Технологический институт Бхилая, \\ Индия, Дург, 491001
}

\begin{abstract}
Аннотация. ЭКГ (электрокардиограмма) представляет собой сигнал в виде комбинации потенциалов, отражающих работу сердца. Эти сигналы часто искажаются мешающими факторами с высокой амплитудой и частотой при сборе, хранении и передаче. Кроме того, эти мешающие факторы могут изменить форму ЭКГ-сигналов и препятствовать получению точных интерпретаций, следовательно, для клинической оценки требуется их соответствующее описание. В данной работе ЭКГ-сигналы из базы данных МIT-BIH характеризируются с помощью метода интерполяционных полиномов. В данном методе сначала выбираются подходящие чебышевские узлы интерполяции в требуемом интервале ЭКГ и эти узлы используются для интерполяции Лагранжа для восстановления ЭКГ-сигнала. В дальнейшем результаты интерполяции улучшаются с помощью сегментирования ЭКГ-сигналов на соответствующее количество сегментов с использованием метода восходящих временных рядов. Эффективность предложенного метода проанализирована с точки зрения стандартных параметров ЭКГ: среднее абсолютное отклонение, корень из среднеквадратичного отклонения, процентная ошибка среднеквадратичной разницы, отношение сигнал-шум и коэффициент корреляции. Полученные результаты сравниваются с результатами существующих методов и показаны преимущества и диагностическая применимость предложенного метода. Также исследованы области применения данного метода.
\end{abstract}

Ключевые слова: ЭКГ-сигнал; восходящий алгоритм; узлы Чебышева; интерполяция Лагранжа-Чебышева; процентное отношение среднеквадратичной разницы; отношение сигнал-шум

\section{ВВЕДЕНИЕ}

ЭКГ (электрокардиография) — это процесс точной записи электрических импульсов, сгенерированных в соответствии функционированием сердца, которая в общем получается с помощью помещения электродов на определенные участки конечностей и грудной клетки. Эти нестационарные сигналы имеют небольшие вариации амплитуды в ограниченном частотном диапазоне и часто искажаются при сбоpe, хранении и передаче мешающими факторами, такими как помехи линий питания (ПЛП), дрейф базы, шум контакта электрода, движение, сокращение мышц, инструментальный шум и др. [1]. Кроме того, эти шумы влияют на характеристики ЭКГ-сигнала, поэтому требуется эффективная характеристика этих сигналов для точной диагностики. Поскольку временная структура этих сигналов является диагностически важной [2], алгоритмы выделения характеристик должны сохранять временные характеристики даже после обработки.

Первый шаг в анализе ЭКГ - удаление шумов. Поскольку шумы, введенные в ЭКГ-сигналы, имеют различные уровни амплитуды и частоты, значительное удаление помех не может быть достигнуто с помощью одного фильтра. Традиционные аналоговые и 


\section{БИБЛИОГРАФИЧЕСКИЙ СПИСОК}

1. Ebrahimzadeh, E.; Pooyan, M.; Jahani, S.; Bijar, A.; Setaredan, S. K. "ECG signals noise removal: Selection and optimization of the best adaptive filtering algorithm based on various algorithms comparison," Biomedical Engineering: Applications, Basis Commun., Vol. 27, No. 4, 1550038, 2015. DOI: 10.4015/S10162372 15500386 .

2. Jha, C. K.; Kolekar, M. H. "ECG data compression algorithm for tele-monitoring of cardiac patients," Int. $J$ Telemedicine and Clinical Practices, Vol. 2, No. 1, p. 31-41, 2017. DOI: 10.1504/IJTMCP.2017.082106.

3. Bhogeshwar, S. S.; Soni, M. K.; Bansal, D. "Design of simulink model to denoise ECG signal using various IIR \& FIR filters," Proc. of Int. Conf. on Reliability Optimization and Information Technology, ICROIT, 6-8 Feb. 2014, Faridabad, India. IEEE, 2014, pp. 477-483. DOI: 10.1109/ICROIT.2014.6798370.

4. Gaikwad, K. M.; Chavan, M. S. "Removal of high frequency noise from ECG signal using digital IIR butterworth filter," Proc. of IEEE Global Conf. on Wireless Computing and Networking, GCWCN, 22-24 Dec. 2014, Lonavala, India. IEEE, 2014, pp. 121-124. DOI: $10.1109 /$ GCWCN.2014.7030861.

5. Sharma, I.; Mehra, R.; Singh, M. "Adaptive filter design for ECG noise reduction using LMS algorithm," Proc. of 4th Int. Conf. on Reliability, Infocom Technologies and Optimization, 2-4 Sept. 2015, Noida,
India. IEEE, 2015, pp. 1-6. DOI: 10.1109/ICRITO.2015. $\underline{7359333 .}$

6. Biswas, U.; Das, A.; Debnath, S.; Oishee, I. "ECG signal denoising by using least-mean-square and normalised-least-mean-square algorithm based adaptive filter," Proc. of 2014 Int. Conf. on Informatics, Electronics \& Vision, ICIEV, 23-24 May 2014, Dhaka, Bangladesh. IEEE, 2014, pp. 1-6. DOI: 10.1109/ICIEV.2014.6850857.

7. Javed, S.; Ahmad, N. A. "An adaptive noise cancelation model for removal of noise from modeled ECG signals," Proc. of IEEE Region 10 Symp., 14-16 Apr. 2014, Kuala Lumpur, Malaysia. IEEE, 2014, pp. 471-475. DOI: $10.1109 /$ TENCONSpring.2014.6863079.

8. Wang, J. S.; Zhang, Y.; Zhang, P.; Sun, S.-F. "Research on denoising algorithm for ECG signals," Proc. of 29th Chinese Control Conf., 29-31 Jul. 2010, Beijing, China. IEEE, 2010, pp. 2936-2940. URI: https://ieeex plore.ieee.org/document/5574064.

9. Peng, Z.; Wang, G. "Study on optimal selection of wavelet vanishing moments for ECG denoising," Sci. Rep., Vol. 7, No. 1, p. 4564, 2017. DOI: 10.1038/s41598017-04837-9.

10. Allami, R.; Stranieri, A.; Balasubramanian, V.; Jelinek, H. F. "A genetic algorithm-neural network wrapper approach for bundle branch block detection," Proc. of Computing in Cardiology Conf., CinC, 11-14 Sept. 2016, Vancouver, Canada. IEEE, 2016, pp. 461-464. DOI: 10.23919/CIC.2016.7868779.

11. Pasolli, E.; Melgani, F. “Genetic algorithm-based method for mitigating label noise issue in ECG signal classification," Biomedical Signal Processing and Control, Vol. 19, p. 130-136, 2015. DOI: 10.1016/j.bspc. 2014.10.013.

12. Kumar, S. S.; Mohan, N.; Prabaharan, P.; Soman, K. P. "Total variation denoising based approach for R-peak detection in ECG signals," Procedia Computer Sci., Vol. 93, p. 697-705, 2016. DOI: 10.1016/j.procs. 2016.07.268.

13. Yadav, O. P.; Ray, S. "Piecewise modeling of ECG signals using Chebyshev polynomials," in: Behera, H. S.; Nayak, J.; Naik, B.; Abraham, A. (eds.) Computational Intelligence in Data Mining. Advances in Intelligent Systems and Computing. Singapore: Springer, Vol. 711, pp.287-296. DOI: 10.1007/978-981-10-8055526.

14. Szegö, G. Orthogonal Polynomials. Vol 23, 4th ed. Rhode Island: AMS, 1939, 1975.

15. Björck, A.; Pereyra, V. "Solution of vandermonde systems of equations," Mathematics Computation, Vol. 24, No. 112, p. 893-903, 1970. URI: https://www.ams.org/ journals/mcom/1970-24-112/S0025-5718-1970-0290541 -1/S0025-5718-1970-0290541-1.pdf.

16. Caporale, G. M.; Cerrato, M. "Chebyshev polynomial approximation to approximate partial differential equations," Working Papers 2008 16, Business School - Economics, University of Glasgow. URI: https://ideas.repec.org/p/gla/glaewp/2008 16.html. 
17. De Villiers, J. Mathematics of Approximation, Vol 1. Springer Science \& Business Media, 2012. DOI: 10.2991/978-94-91216-50-3.

18. Kwan, B. H.; Ong, K. M.; Paramesran, R. "Noise removal of ECG S signals using Legendre moments," Proc. of 27th Annual Int. Conf. on Engineering in Medicine and Biology Society, 2005. IEEE, 2005, pp. 5627-5630. DOI: $10.1109 /$ IEMBS.2005.1615762.

19. Powell, M. J. "On the maximum errors of polynomial approximations defined by interpolation and by least squares criteria," The Computer J., Vol. 9, No. 4, p. 404-407, 1967. DOI: 10.1093/comjn1/9.4.404.

20. Brisebarre, N.; Joldeş, M. "Chebyshev interpolation polynomial-based tools for rigorous computing," Proc. of 2010 Int. Symp. on Symbolic and Algebraic Computation. ACM, 2010, pp. 147-154.

21. Goldberger, A. L.; Amaral, L. A.; Glass, L.; Hausdorff, J. M.; Ivanov, P. C.; Mark, R. G.; Mietus, J. E.; Moody, G. B.; Peng, C. K.; Stanley, H. E. "PhysioBank, physioToolkit, and physioNet: components of a new research resource for complex physiologic signals," Circulation, Vol. 101, No. 23, p. 215-220, 2000. PMID: 10851218.

22. Sandryhaila, A.; Saba, S.; Puschel, M.; Kovacevic, J. "Efficient compression of QRS complexes using Hermite expansion," IEEE Trans. Signal Processing, Vol. 60, No. 2, p. 947-955, 2012. DOI: 10.1109/TSP.2011. 2173336.

23. Ouali, M. A.; Ghanai, M.; Chafaa, K. "Upper envelope detection of ECG signals for baseline wander correction: a pilot study," Turkish J. Electrical Engineering \& Computer Sciences, Vol. 26, No. 2, p. 803-816, 2018. DOI: $\underline{10.3906 / \mathrm{elk}-1705-165}$.

24. Jokic, S.; Delic, V.; Peric, Z.; Krco, S.; Sakac, D. "Efficient ECG modeling using polynomial functions," Elektronika ir Elektrotechnika, Vol. 110, No. 4, p. 121-124, 2011. DOI: $10.5755 /$ j01.eee.110.4.304.

25. Xavier, B.; Dahikar, P. B. "Iterative least square polynomial approximation method for filtering ECG signals," European J. Advances in Engineering and Technology, Vol. 3, No. 7, p. 65-70, 2016. URI: http:// www.ejaet.com/PDF/3-7/EJAET-3-7-65-70.pdf.

26. Nahiyan, K. T.; Al Amin, A. "Removal of ECG baseline wander using Savitzky-Golay filter based method," Bangladesh J. Medical Physics, Vol. 8, No. 1, 2015. DOI: $10.3329 /$ bjmp.v8i1.33932.

27. Kwan, B.-H.; Ong, K.-M.; Paramesran, R. "Noise removal of ECG signals using Legendre moments," Proc. of IEEE 27th Annual Int. Conf. on Engineering in Medici- ne and Biology Society, 17-18 Jan 2006, Shanghai, China. IEEE, 2006, pp. 5627-5630. DOI: 10.1109/IEMBS.2005. 1615762 .

28. Castiglioni, P.; Piccini, L.; Di Rienzo, M. "Interpolation technique for extracting features from ECG signals sampled at low sampling rates," Proc. of Conf. on Computers in Cardiology, 21-24 Sept. 2003, Thessaloniki Chalkidiki, Greece. IEEE, 2003, pp. 481-484. DOI: 10.1109/CIC.2003.1291197.

29. Gacek, A.; Pedrycz, W. ECG Signal Processing, Classification and Interpretation: A Comprehensive Framework of Computational Intelligence. Springer Science \& Business Media, 2012. DOI: 10.1007/9780-85729-868-3.

30. Yadav, O. P.; Ray, S. "Smoothening and segmentation of ECG signals using total variation denoising-minimization-majorization and bottom-up approach," Procedia Computer Science, Vol. 85, p. 483-489, 2016. DOI: 10.1016/j.procs.2016.05.195.

31. Ray, S.; Yadav, O. P. "Approximation of ECG signals using Chebyshev polynomials," Proc. of 7th Int. Workshop on Reliable Engineering Computing with Polymorphic Uncertain Data, Ruhr University Bochum, Germany, vol. 15, pp. 335-348, 2016.

32. Burns, A.; Greene, B. R.; McGrath, M. J.; O'Shea, T. J.; Kuris, B.; Ayer, S. M.; Stroiescu, F.; Cionca, V. "Shimmer - a wireless sensor platform for noninvasive biomedical research," IEEE Sensors J., Vol. 10, No. 9, p. 1527-1534, 2010. DOI: 10.1109/JSEN.2010. 2045498.

33. Alhelal, D.; Aboalayon, K. A. I.; Daneshzand, M.; Faezipour, M. "FPGA-based denoising and beat detection of the ECG signal," Proc. of 2015 IEEE Long Island Systems, Applications and Technology Conf., LISAT, 1 May 2015, Farmingdale, USA. IEEE, 2015, pp. 1-5. DOI: 10.1109/LISAT.2015.7160184.

34. Anapagamini, S.; Rajavel, R. "Hardware implementation of ECG denoising system using TMS320C6713 DSP processor," Int. J. Biomedical Engineering Technology, Vol. 21, No. 1, p. 95-108, 2016. DOI: $10.1504 /$ IJBET.2016.076735.

35. Romero, V. B.; Atienza, D.; Khaled, N. "ECG baseline wander removal and noise suppression analysis in an embedded platform," Proyecto Fin de Master en Ingenierı de Computadores, Master en Investigacion en Informatica, Facultad de Informatica, Universidad Complutense de Madrid, 2009. URI: https://core.ac.uk/ download/pdf/19712998.pdf. 